\title{
Design and Modelling of a Cable-Driven Parallel-Series Hybrid Variable Stiffness Joint Mechanism for Robotics
}

\author{
Cihat Bora Yigit and Pinar Boyraz \\ Department of Mechanical Engineering, Istanbul Technical University, Inonu Cd. No:65, 34437, Beyoglu, \\ Istanbul, Turkey
}

Correspondence to: Cihat Bora Yigit (yigitci@itu.edu.tr) and Pinar Boyraz (pboyraz@itu.edu.tr)

Received: 25 November 2016 - Revised: 12 February 2017 - Accepted: 24 February 2017 - Published: 22 March 2017

\begin{abstract}
The robotics, particularly the humanoid research field, needs new mechanisms to meet the criteria enforced by compliance, workspace requirements, motion profile characteristics and variable stiffness using lightweight but robust designs. The mechanism proposed herein is a solution to this problem by a parallel-series hybrid mechanism. The parallel term comes from two cable-driven plates supported by a compression spring in between. Furthermore, there is a two-part concentric shaft, passing through both plates connected by a universal joint. Because of the kinematic constraints of the universal joint, the mechanism can be considered as a serial chain. The mechanism has 4 degrees of freedom (DOF) which are pitch, roll, yaw motions and translational movement in $z$ axis for stiffness adjustment. The kinematic model is obtained to define the workspace. The helical spring is analysed by using Castigliano's Theorem and the behaviour of bending and compression characteristics are presented which are validated by using finite element analysis (FEA). Hence, the dynamic model of the mechanism is derived depending on the spring reaction forces and moments. The motion experiments are performed to validate both kinematic and dynamic models. As a result, the proposed mechanism has a potential use in robotics especially in humanoid robot joints, considering the requirements of this robotic field.
\end{abstract}

\section{Introduction}

In recent years, there has been an emergent need in robotics to develop new mechanisms that go beyond the conventional structures, focusing on compliant, lightweight and energy efficient designs. Following this need, increasing number of studies related with non-conventional robot mechanism design are reported (Grioli et al., 2015). In addition, large workspace, smooth motion profiles, and new mechanical structures with certain redundancies to ease the control applications can be considered as desired properties of such mechanisms. Mizuuchi et al. (2002), Yang et al. (2005), Ham et al. (2009) and Vanderborght et al. (2013) also emphasizes that such joint designs are needed in robotics instead of conventional structures.

Most of non-conventional mechanisms are studied in continuum and hyper-redundant robotics and used especially in minimally invasive surgery (MIS) robotics. These structures combine the compliancy and lightweight compact design requirements, although they may fall short of controllability and introduce more complexity in modelling. For instance, in Gravagne et al. (2003) a planar continuum robot is introduced accounting the large deflection dynamics, examining the dynamics of a planar backbone section. Another approach for designing compliant and lightweight design can be seen in Wendlandt and Sastry (1994), employing a parallel kinematic mechanism (PKM) together with a spring and a spherical joint in the middle. Instead of including PKM or spring, a typical approach is to propose multi-sections as given in Jones and Walker (2006) in order to increase the controllability of the mechanical structure. Additionally, a PKM mechanism, called cable-driven universal joint (CPUJ), is presented as a module (Lim et al., 2009) and multisection properties are also examined in Lim et al. (2012). These mechanisms are used widely in continuum robotics, although satisfying most of the requirements that come with the disadvantage of complex dynamics. One way to overcome the problem of complex and highly-nonlinear dynamics, spatial models considering the large deformation are used (Tunay, 
2013). On the other hand, an extra difficulty comes from the compactness requirements. Since the continuum backbone structures have often small diameter in nature, there is very limited space to fit the actuation units within the module. Therefore, a cable-driven remote manipulation is preferred with often an antagonist arrangement (Potkonjak et al., 2011). Although, it can be used for a different purpose, a very similar humanoid neck mechanism is proposed in Gao et al. (2012b) and is based on a compression spring and two parallel plates, driven by four cables. The same research group also studied inverse kinematics of the structure in Gao et al. (2012a); however, it differs from the mechanism proposed in this work since in their mechanism, there is no serial link to restrict the highly complex spring movement. A similar mechanism is introduced in Nori et al. (2007) and a partial kinematic model with control strategy is presented. In order to handle the nonlinear dynamics of continuum robots, the elastic rod dynamic behavior can be taken as a model using Cosserat Theory in Cao and Tucker (2008). The continuum robotics literature is diverse in terms of pointing the new directions in mechanism design and a detailed review can be found in Walker (2013). However, there are still alternatives to elastic back-bone and continuum structures that may lead to more feasible structures. For example novel 3 DOF fully parallel manipulators with rotational capabilities which is given in Liu et al. (2005) can also be considered as good candidates for especially humanoid neck and joint design. The alternative multi-section designs can be structures such as given in Woehrmann et al. (2013) with effective magnetic actuation and interleaved continuum-rigid manipulators as presented in Conrad et al. (2013). Moreover, a non-compliant but similar structure to the mechanism proposed in this paper, known as 3-SPS mechanism is studied in Alici and Shirinzadeh (2004) and Kim et al. (2015).

In addition to given studies in continuum robotics and cable-driven robotic mechanisms, robotic joint with varying stiffness/compliance is also required. In the last decade, many inspiring developments occured in this area. In Ham et al. (2009) and Vanderborght et al. (2013), the most important ones are summarized and classified. Among this classification, the structure-controlled stiffness in Ham et al. (2009) uses the natural characteristics of the elastic element providing the compliance. The "Jack Spring" mechanism in Hollander et al. (2005) controls the number of active coils by using a screw mechanism to adjust the stiffness without using any additional elements in the mechanism, which simplifies the design. The proposed mechanism in this paper can be included in this class since it uses natural mechanical behaviour of the helical spring under bending and compression effects.

In this study, a cable-driven, compression springsupported hybrid mechanism is proposed. The advantages of the proposed mechanism are the unique combination of multiple traits of variable compliancy, hybrid parallel-serial structure for better controllability and lightweight design.
The mechanism can be used in robotic joints especially in humanoid design due to its smooth motion profile, its potential in design of a multi-section robot as a module or section which is individually controllable. The main contribution of this paper is to present a new joint mechanism design which combines advantages of two different joint design approaches. The first approach, which is given in Nori et al. (2007) and Gao et al. (2012b), is to use compression spring and cable-driven actuation. The most important disadvantage of these structures is modelling and control difficulties. These mechanisms do not have accurate mathematical models as serial manipulators have. In the proposed mechanism, the additional shaft and the universal joint constrains the motion, which in turn allows treating it as a serial mechanism and facilitates calculations of the kinematic and dynamic modelling which are also presented. Furthermore, the shaft allows transferring yaw motion directly. In the second approach, Yang et al. (2005), Lim et al. (2009, 2012), Alici and Shirinzadeh (2004) and Kim et al. (2015) use the two part shaft and the universal joint however the absence of compression spring results in a stiff structure. On the other hand, proposed mechanism shows a compliant behaviour as a result of the additional translation motion and the helical compression spring. Linear helical compression spring provides nonlinear stiffness characteristics under combined bending and compression effects. Besides, nonlinear stiffness characteristics is essential for a variable stiffness actuator design and most of the designs make use of complex nonlinear spring mechanisms in Ham et al. (2009) and Vanderborght et al. (2013). Another contribution of this study to design literature on variable stiffness actuators is the simplification of nonlinear stiffness mechanism. Therefore, the helical spring can be assumed as nonlinear stiffness mechanism under compression and bending effects. Although it is a commonly used machine element, there exist few studies analysing combined effect of bending and compression on a helical spring. In Leech (1994), shape memory alloy wires are used as actuators and two different spring loading scenarios are analysed. First a single-sided load is applied, second a pure bending is analysed. Both solutions are based on Castigliano's Theorem. In this study, the same theorem is used and the solution is improved numerically with the combination of bending and compression scenario.

This paper first presents the main ideas in the concept of cable-driven parallel-series hybrid mechanism (CDPS) in Sect. 2. Then, the kinematic modelling is presented in Sect. 3. Next, in Sect. 4, the full dynamic modelling of this hybrid mechanism is reported. In Sect. 5, both experimental structure and results are detailed. Finally, in Sect. 6, the conclusions are drawn and further work on the mechanism design are proposed. 


\section{Description of Cable-Driven Parallel-Series (CDPS) Hybrid Mechanism}

The proposed mechanism includes a lower plate and an upper plate which are used to hold the compression spring in concentric position as shown in Fig. 1. The structure is driven by three cables pulled or set free by three servo motors located underneath the CDPS mechanism. The upper plate is able to perform rotation in two axes providing roll and pitch angles. This configuration is further supported by a concentric shaft with a universal joint in the middle, which passes through the mechanism restricting and defining the bending motion of the compression spring. The concentric shaft is actuated by a fourth motor to provide the movement about $z$ axis yaw angle. These four motors are encapsulated in a separate case and can be installed at a far location from the mechanism which makes the mechanism lightweight and remotelyactuated. Despite the fact that the mechanism can be classified as a parallel mechanism because of its main construction properties, it is modelled as a serial mechanism due to serial kinematics imposed by the middle shaft and the universal joint. Therefore, the mechanism is called as CDPS hybrid mechanism.

The mechanism has $4 \mathrm{DOF}$ in total, 3 of them are related with motion and the other one is a translational motion along $z$ axis for adjusting the stiffness. Two rotations of upper plate (roll and pitch) are actuated in a cable-driven way, however the motion is constrained by the shaft inside the spring and the universal joint. The restriction in the motion simplifies the kinematic and dynamic calculations. The last DOF of the mechanism is the translational motion of the upper plate along $z$ axis and is designed to adjust the stiffness value of the spring which determines the combined stiffness of the mechanism in pitch and roll axes. Within this study, the control is achieved over stiffness via a structure-dependent way without using additional screw mechanism as in Hollander et al. (2005). The yaw motion herein is not inherently compliant, therefore modelling and experiments sections do not include yaw motion in this work and examine other 3 DOF (pitch, roll and translation). However, when required, the compliant behavior can be induced using a torsional spring at the lower plate.

\section{Kinematic Modelling}

The upper and lower plates of the CDPS mechanism are connected by a shaft with a universal joint. Therefore, excluding the yaw motion, the neck mechanism is essentially considered as a serial manipulator with rotation around two different axes at the center of the shaft such as pitch and roll and a translation at the end of the shaft, summing up to $3 \mathrm{DOF}$. The shaft having the universal joint in the middle of the structure transmits the torque to change the yaw angle while providing a geometric constraint for resolving the spring forces because it determines the bending point. Therefore, in this mechanism

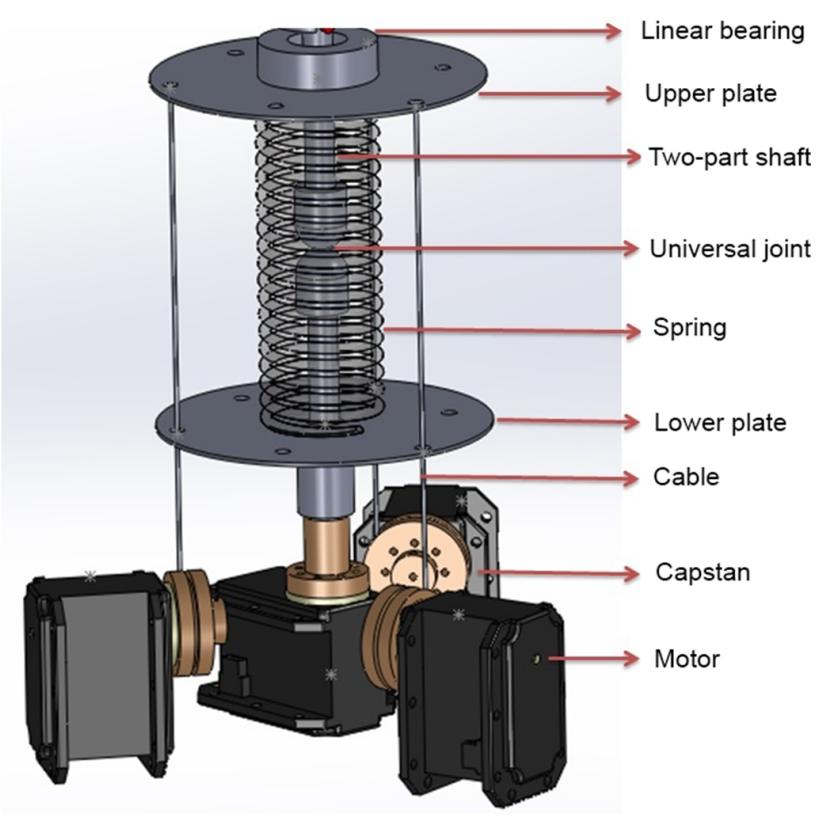

Figure 1. CDPS mechanism and its components, with motors, bearings and capstans.

the amount of the compression can be taken into account and be described by distance between the center of the universal joint and the upper plate. The yaw motion of shaft does not affect the upper plate, because it is directly transmitted to the robotic head platform. The pitch and roll $(\theta, \phi)$ angles of the upper plate are determined by the cable lengths. Therefore, the relative position of the upper plate with reference to the lower plate can be defined by using three generalised coordinates $\left(\theta, \phi, d_{2}\right)$. They are included in a vector which is denoted as $\boldsymbol{q}$, noting that the vectors are shown with bold italic characters. The variable $d_{2}$ represents the distance between the center of the universal joint and the upper plate. The geometric variables used in deriving the kinematic model are given schematically in Fig. 2.

It is clearly seen in Fig. 3 that the neck mechanism is an RRP structure with $3 \mathrm{DOF}$, having the variables roll, pitch and translation $\left(\theta, \phi, d_{2}\right)$. The local frames $\left(\boldsymbol{X}_{1}, \boldsymbol{Y}_{1}, \boldsymbol{Z}_{1}\right)$ and $\left(\boldsymbol{X}_{2}, \boldsymbol{Y}_{2}, \boldsymbol{Z}_{2}\right)$ intersect and one of them is rotated by angle $\pi / 2$ with respect to the other one. The distance $d_{1}$ is constant since it is structurally fixed.

Having defined the variables of the kinematic model, now one can formulate and solve the forward and inverse kinematics. In forward kinematics, the cable lengths $\left(l_{1}, l_{2}, l_{3}\right)$ or the motor shaft angles are inputs and the roll, pitch angles and translation of the upper plate $\left(\theta, \phi, d_{2}\right)$ are the outputs. On the other hand, in inverse kinematics, $\left(\theta, \phi, d_{2}\right)$ are inputs and the cable lengths or necessary motor shaft angles are outputs. In order to derive the kinematics, we can use transformation between the upper plate and the lower plate. Since the points where the cables are attached on the upper plate $\left(\boldsymbol{P}_{1}, \boldsymbol{P}_{2}, \boldsymbol{P}_{3}\right)$ can define the upper plane and the points 


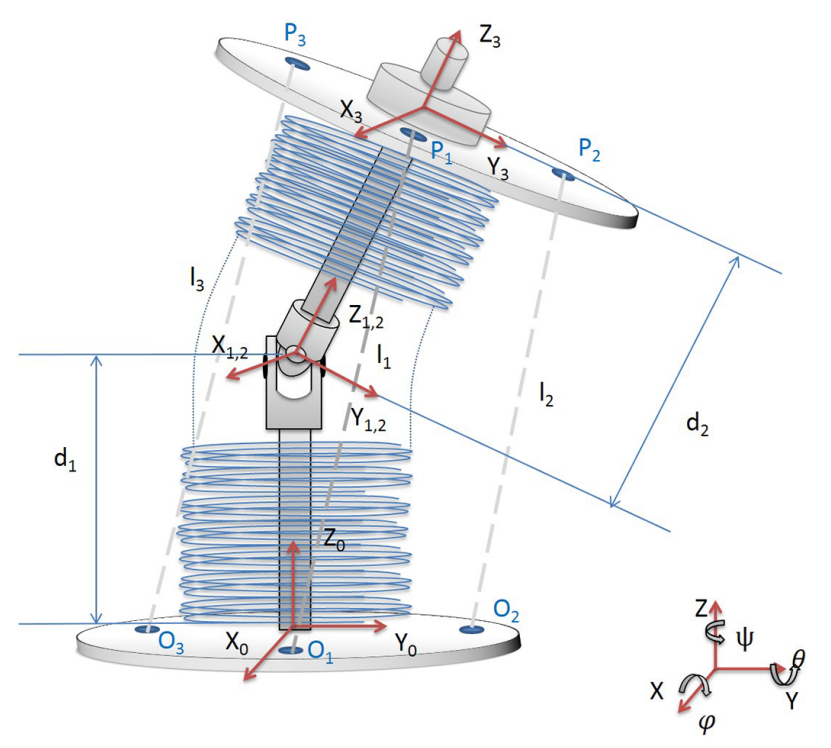

Figure 2. Neck mechanism with variables, local and global axes, cable lengths, upper and lower plate cable assembly points.

where the cables pass through at the lower plate can define the lower plane $\left(\boldsymbol{O}_{1}, \boldsymbol{O}_{2}, \boldsymbol{O}_{3}\right)$, the transformation matrix is used to define $\boldsymbol{P}_{i}$ according to $\boldsymbol{O}_{i}$. The translation $\left(\boldsymbol{T r}_{1}\right)$ of constant length of the lower part of the shaft $\left(d_{1}\right)$, the rotations ( $\left.\boldsymbol{R o t}_{1}\right)$ of pitch and roll $(\theta, \phi)$ and the translation $\left(\mathbf{T r}_{2}\right)$ of the upper plate $\left(d_{2}\right)$ are taken into account in order to obtain this transformation. These transformations are given in Eq. (1), all together forming the transformation matrix $\mathbf{T}_{03}$ in Eq. (2).

$\mathbf{T r}_{1}=\left[\begin{array}{cccc}1 & 0 & 0 & 0 \\ 0 & 1 & 0 & 0 \\ 0 & 0 & 1 & d_{1} \\ 0 & 0 & 0 & 1\end{array}\right]$

$\boldsymbol{R o t}_{1}=\left[\begin{array}{cccc}c \theta & s \theta s \phi & s \theta c \phi & 0 \\ 0 & c \phi & -s \phi & 0 \\ -s \phi & c \theta s \phi & c \theta c \phi & d_{1} \\ 0 & 0 & 0 & 1\end{array}\right]$

$\mathbf{T r}_{2}=\left[\begin{array}{cccc}1 & 0 & 0 & 0 \\ 0 & 1 & 0 & 0 \\ 0 & 0 & 1 & d_{2} \\ 0 & 0 & 0 & 1\end{array}\right]$

$\mathbf{T}_{03}=\left[\begin{array}{cccc}c \theta & s \theta s \phi & s \theta c \phi & s \theta c \phi d_{2} \\ 0 & c \phi & -s \phi & -s \phi d_{2} \\ -s \phi & c \theta s \phi & c \theta c \phi & c \theta c \phi d_{2}+d_{1} \\ 0 & 0 & 0 & 1\end{array}\right]$

Then, the transformation matrix is used to calculate $\boldsymbol{P}_{i}=$ $\left[P_{i, x}, P_{i, y}, P_{i, z}\right]^{\mathrm{T}}$ using $\boldsymbol{O}_{i}=\left[O_{i, x}, O_{i, y}, O_{i, z}\right]^{\mathrm{T}}$ via Eq. (3).

$\left[\begin{array}{c}\boldsymbol{P}_{i} \\ 1\end{array}\right]=\mathbf{T}_{03}\left[\begin{array}{c}\boldsymbol{O}_{i} \\ 1\end{array}\right]$

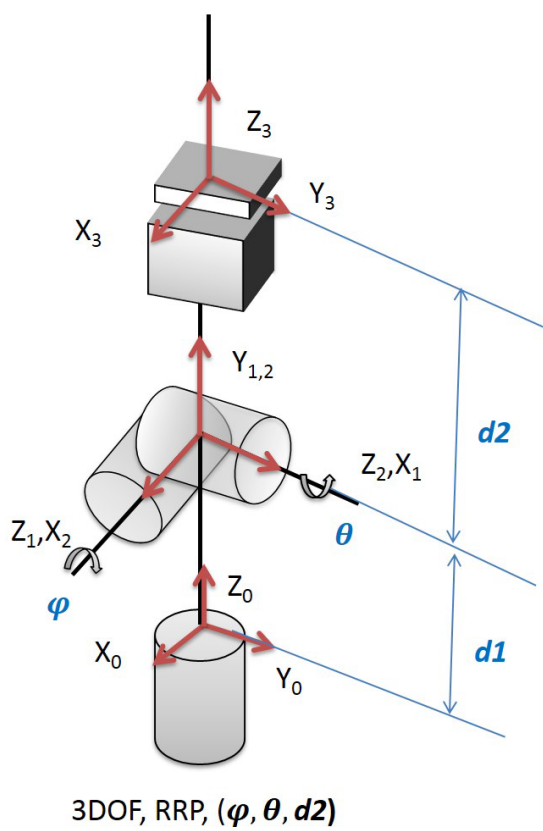

Figure 3. Reduced serial kinematic model of neck mechanism with RRP structure (pitch, roll and translation).

In order to relate the cable lengths $\left(l_{1}, l_{2}, l_{3}\right)$ with upper plate position $\left(\theta, \phi, d_{2}\right)$, the definition of the Euclidean distance between $\boldsymbol{O}_{i}$ and $\boldsymbol{P}_{i}$ is used. According to this definition, any cable length can be defined by the expression given in Eq. (4).

$l_{i}=\sqrt{\left(P_{i, x}-O_{i, x}\right)^{2}+\left(P_{i, y}-O_{i, y}\right)^{2}+\left(P_{i, z}-O_{i, z}\right)^{2}}$,

where $i=1,2,3$

If we could obtain the correct $\boldsymbol{P}_{i}$ coordinates using $\boldsymbol{O}_{i}$ from the kinematic solution, the nonlinear error function $f_{i}$ defined by Eq. (5) must be zero.

$$
\begin{aligned}
f_{i} & =l_{i}-\sqrt{\left(P_{i, x}-O_{i, x}\right)^{2}+\left(P_{i, y}-O_{i, y}\right)^{2}+\left(P_{i, z}-O_{i, z}\right)^{2}} \\
& =0
\end{aligned}
$$

Using Eqs. (3) and (5), any variable can be obtained numerically using recursive Newton-Raphson algorithm given by Eq. (6).

$\boldsymbol{q}_{k}=\boldsymbol{q}_{k-1}-\mathbf{J}^{-1} \boldsymbol{F}$

where $\mathbf{J}$ is the Jacobian matrix defined by Eq. (7) and $\boldsymbol{q}_{k}$ is the $k$ th iteration of the solution for the vector of the generalised coordinates, while vector $\boldsymbol{F}$ is formed by equations $f_{i}$. 
$\mathbf{J}=\left[\begin{array}{lll}\frac{\partial f_{1}}{\theta} & \frac{\partial f_{1}}{\phi} & \frac{\partial f_{1}}{d_{2}} \\ \frac{\partial f_{2}}{\theta} & \frac{\partial f_{2}}{\phi} & \frac{\partial f_{2}}{d_{2}} \\ \frac{\partial f_{3}}{\theta} & \frac{\partial f_{3}}{\phi} & \frac{\partial f_{3}}{d_{2}}\end{array}\right]$

The solution of inverse kinematics is straightforward by using Eq. (5) when desired state variables are known. Additionally, a simple kinematic model is included, which minimizes the compression of the spring. The model assumes one cable is fixed at the initial position and takes only an orientation input. It is indeed possible to realize all the pitch and roll angles by manipulating only two cables. For each cable, a separate solution loop is defined according to the selected non-moving (i.e. idle) cable. The solution proceeds in the appropriate direction (i.e. towards minimizing approximation error) for finding the lengths of the remaining cables to realize the roll and pitch angles given in the inverse kinematic problem. According to this method, only two selected cables are manipulated at any motion command. This approach is also reflected at the simplified Jacobian matrix used in the inverse kinematic problem as given in Eq. (8).

$\mathbf{J}=\left[\begin{array}{lll}\frac{\partial f_{1}}{l_{2}} & \frac{\partial f_{1}}{l_{3}} & \frac{\partial f_{1}}{d_{2}} \\ \frac{\partial f_{2}}{l_{2}} & \frac{\partial f_{2}}{l_{3}} & \frac{\partial f_{2}}{d_{2}} \\ \frac{\partial f_{3}}{l_{2}} & \frac{\partial f_{3}}{l_{3}} & \frac{\partial f_{3}}{d_{2}}\end{array}\right]=\left[\begin{array}{lll}0 & 0 & \frac{\partial f_{1}}{d_{2}} \\ 1 & 0 & \frac{\partial f_{2}}{d_{2}} \\ 0 & 1 & \frac{\partial f_{3}}{d_{2}}\end{array}\right]$

The simplified kinematic model can be better understood by looking at the Fig. 4 where top view of the lower plate is given. The virtual lines between the points of $O_{i}$ and the center divides the plate into three areas. Desired pitch and roll motions can be shown as a vector to determine the moving and fixed cables. Two cables neighbouring the area which includes the motion vector, are pulled to perform the desired motion. Besides, opposite cable length is set to default initial value. For example, to complete a $30^{\circ}$ rotation in both pitch and roll axes, the distance between upper plate and the lower plate has to be decreased in the direction of vector $\boldsymbol{E}$. Thus, the neighbouring cables of this area which includes the vector are cable 1 and 3 . The amount of pull or motor shaft rotation for these cables are calculated by using Eqs. (7) and (8). The length of cable 2 is set to default value.

The mechanism is implemented as a humanoid neck platform and it is used throughout the study. The same parameters with the implemented mechanism, which are given in Table 1, are used for workspace simulation. The positions of the midpoint and the orientations of the upper plate are calculated, using simplified forward kinematics, i.e., keeping one cable length constant at default value $(95 \mathrm{~mm})$ while changing the other two within 50-95 mm range, as seen in Fig. 5.

The workspace for the simplified kinematic algorithm is given in Fig. 5a. The workspace is obtained as a sum of three distinct cases. In each case, one of the cable is held at $95 \mathrm{~mm}$. The midpoint of the upper plate is shown with different colours and symbols for each case. Figure $5 b$ and $c$

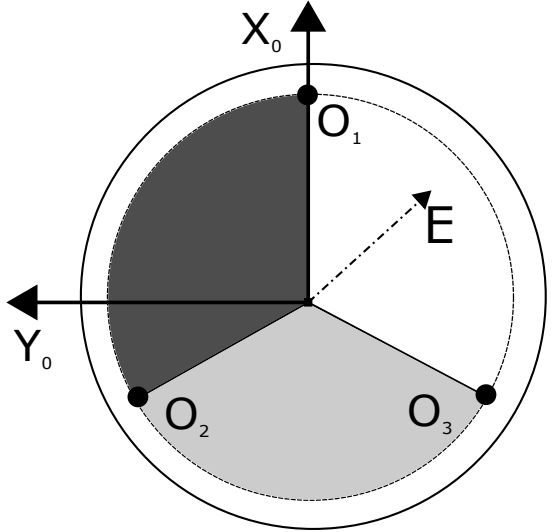

Figure 4. Top view of the lower plate and an example motion command vector.

Table 1. Simulation parameters in workspace analysis.

\begin{tabular}{lr}
\hline Variable & Value [unit] \\
\hline Lower shaft length $d_{1}$ & $47[\mathrm{~mm}]$ \\
Length of constant cable & $95[\mathrm{~mm}]$ \\
Distance between lower plate center and $O_{i}$ points & $40[\mathrm{~mm}]$ \\
Distance between upper plate center and $P_{i}$ points & $40[\mathrm{~mm}]$ \\
\hline
\end{tabular}

show $Y-Z$ and $X-Z$ views of the same figure, respectively. The cable numbers 2 and 3 are placed symmetrically with respect to $x$ axis which results a symmetrical distribution in $Y-Z$ plane. Since the cable number 1 is on the $x$ axis and there is no cable on the opposite side, the midpoints are distributed asymmetrically in $X-Z$ plane. Thus, single roll motions require at least two cables ( 2 and 3 ) have to be pulled. Even though, single positive pitch motion requires action of two cables similar to roll motion, only cable 1 is responsible for single negative roll motions. Figure $5 \mathrm{~d}$ shows the range of motion in pitch and roll angles with respect to given variations of cable lengths. Obviously, each orientation value given in the range of motion (between -30 and $30^{\circ}$ ) is obtained by holding one cable length constant at default value.

Although, the range of motion (ROM) of the human neck differs from person to person regarding to their ages, genders and physical attributes, average values for pitch (flexionextension), roll (lateral bending) and yaw (axial rotation) axes are reported around 60,40 and $80^{\circ}$, respectively in the study of Ferrario et al. (2002). On the other hand, Bennett et al. (2002) shows that only a limited amount of the range (between 30 and $50 \%$ ) are utilised to complete daily tasks. The workspace of the humanoid neck platform is calculated between -30 and $30^{\circ}$ yielding $60^{\circ}$ in total for pitch and roll motions because of practical reasons such as strength of cables and stall torque of motors. Additionally, the lower plate blocks the upper plate when roll and pitch angles are increased to a certain value which is related with the plate dimensions. Thus, the main drawback of the mechanism can be 
(a)

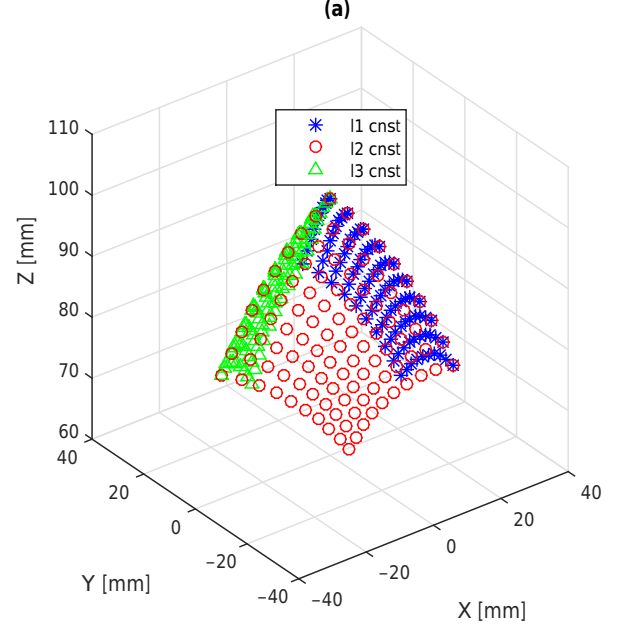

(b)

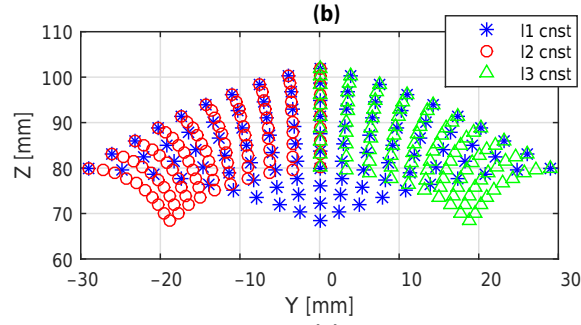

(c)

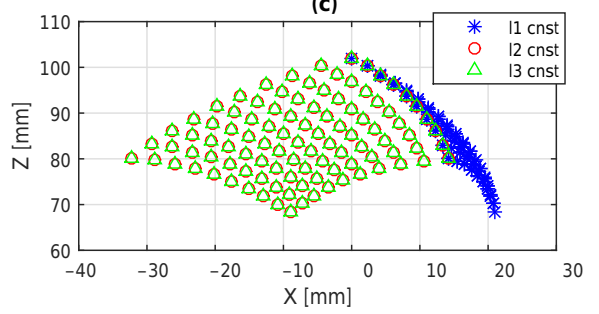

(d)

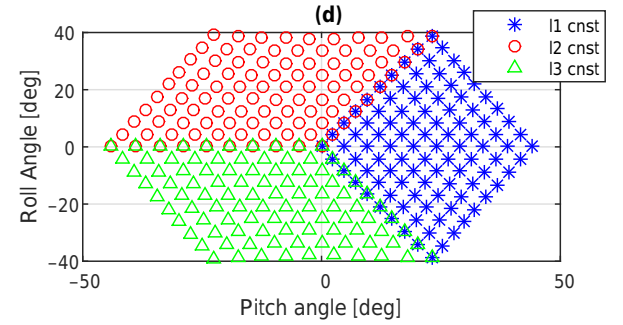

Figure 5. Workspace of the proposed mechanism in (a) 3-D, (b) $Y-Z$, (c) $X-Z$ view, (d) Pitch and Roll Angles.

considered as the motion boundaries when compared to the full ROM of humans. However, it is enough for performing daily activities when the proposed mechanism is used as a humanoid neck joint.

\section{Dynamic Modelling}

In dynamic modelling, the result of kinematic model (position, velocity and acceleration) obtained in Sect. 3 is used to define the dynamic motion of the mechanism under cable based forces. The dynamic modelling is composed of three sequential steps: (i) reduction of 3-D model into a 2-D model without information loss, (ii) force analysis on a bending helical spring using Castigliano's Theorem, and (iii) complete dynamic model.

\subsection{Dimension Reduction of the Model}

To decrease the complexity of computations and its cost during the analysis of helical spring under bending and compression effects, it is possible to reduce the dimension without information loss. Deformations of the helical spring can be considered and calculated in a 2-D model because of its cylindrical shape. In order to simplify the computation of bending of helical spring in 3-D coordinates, a different Euler angles convention is used as follows: Two rotations of mechanism around $\boldsymbol{X}_{0}$ and $\boldsymbol{Y}_{0}$ axes are defined in Euler $(X-Y-Z)$ convention and called roll and pitch angles, re- spectively. However, these two angles can be defined in $\mathrm{Eu}-$ ler $(Z-X-Y)$ convention. Thus, the $X-Y$ plane is rotated around $\boldsymbol{Z}_{0}$ axis so that bending of the helical spring appears only in this plane. Then the bending or rotation around new $x$ axis is called as deflection angle and denoted with $\rho$.

\subsection{Helical Spring Analysis}

The helical spring is subjected to bending effects rather than buckling. The cables are assumed to have constant lengths, hence no plastic/elastic deformation are allowed for the cables. The universal joint in the middle determines the bending point of the helical spring. Figure 6 shows geometrical relations and frames which are used to calculate the dynamic model of the system.

The frame $x_{k} y_{k} z_{k}$ is attached to the bottom center all of the coils. The variable $k$ is the index number of the coils so that $x_{0} y_{0} z_{0}$ and $X_{0} Y_{0} Z_{0}$ are coincident on the lower plate. Successive coil frames are rotated around their $x$ axes in equally so that $z_{n-1}$ and $\boldsymbol{Z}_{3}$ are tangent. The vector $\boldsymbol{N}_{k}$ stands for the origins of all coils.

The same idea is used for helical spring analysis as in Leech (1994) and each coil of the spring is separately analysed. In order to use Castigliano's Theorem, infinitesimal elements are defined in the helical spring. Figure 6 shows the helical spring and the frame definitions. Angular position of the infinitesimal elements on $k$ th coil is defined as $\alpha$ on $x_{k} y_{k}$ plane. 

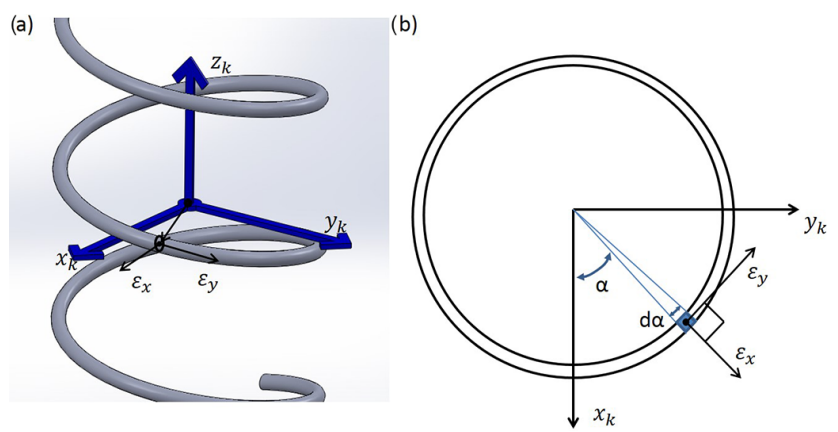

Figure 6. (a) Local frames on spring and infinitesimal element. (b) Top view of a spring coil.

In order to define strength properties of the infinitesimal element on the spring coils, a 2-D frame is attached to all elements. The vector $\boldsymbol{\epsilon}_{x}$ is placed on tangential direction and the vector $\boldsymbol{\epsilon}_{y}$ is placed on normal direction. This frame and the other variables which are used to calculate the deformation of the spring are shown in Fig. 6.

The cable forces are shown in Fig. 7 which are acting on the upper plate are defined as $\boldsymbol{T}_{1}, \boldsymbol{T}_{2}$ and $\boldsymbol{T}_{3}$. These forces are unidirectional and are reduced to generalized forces definition which includes a force on $-z_{n-1}$ direction which is $\boldsymbol{F}_{\text {eq }}$ and a moment around $\boldsymbol{x}_{n-1}$ axis which is $\boldsymbol{M}_{\text {eq }}$. To calculate the relation between the deformation of the spring and the generalized forces, the distances between upper plate center point and central points of the infinitesimal elements have to be defined. The position vector of the central point of the upper plate $P_{0}$ with respect to frame $k$ is represented as ${ }^{k} \boldsymbol{r}_{P_{0}}$. The position of an infinitesimal element " $\mu$ ' on $k$ th coil of the spring, ${ }^{k} \boldsymbol{r}_{\mu}$, is defined as in Eq. (9),

${ }^{k} \boldsymbol{r}_{\mu}={ }^{k} \boldsymbol{r}_{\alpha}+{ }^{k} \boldsymbol{r}_{P_{0}}$

where ${ }^{k} \boldsymbol{r}_{\alpha}$ is described as the position of the infinitesimal element in the particular coil and is given in Eq. (10).

${ }^{k} \boldsymbol{r}_{\alpha}=\left[\begin{array}{c}\mathrm{R} \cos (\alpha) \\ \mathrm{R} \sin (\alpha) \\ \left(N_{k, z}+\frac{N_{k+1, z}-\left(N_{k, z}\right) \alpha}{2 \Pi}\right.\end{array}\right]$

The moment vector, ${ }^{k} \boldsymbol{M}_{\mu}$, which is resulting from the equivalent force vector and is acting on the element $\mu$ is calculated by using cross product given in Eq. (11).

${ }^{k} \boldsymbol{M}_{\mu}={ }^{k} \boldsymbol{r}_{\mu} \times{ }^{k} \boldsymbol{F}_{\mathrm{eq}}$

Next, the total moment acting on the element $\left({ }^{k} \boldsymbol{M}_{\mu \mathrm{t}}\right)$ is calculated as given in Eq. (12).

${ }^{k} \boldsymbol{M}_{\mu \mathrm{t}}={ }^{k} \boldsymbol{M}_{\mu}+{ }^{k} \boldsymbol{M}_{\mathrm{eq}}$

In order to use the moment in Castigliano's Formula, it has to be defined in the element specific frame $\epsilon_{x} \epsilon_{y}$. Since the

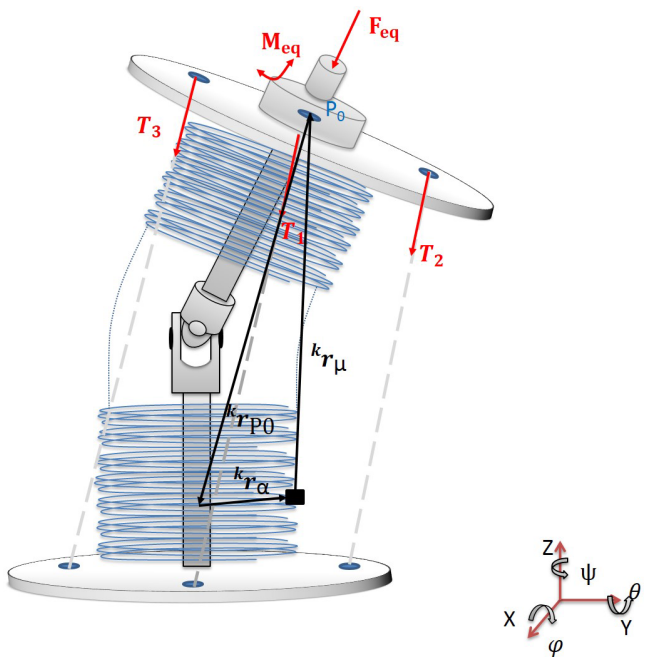

Figure 7. Spring and cable forces acting on the mechanism.

given moments are defined in coil frame, a rotation is needed as $\alpha$ about $z_{k}$ axis, using the rotation matrix $\left(\operatorname{Rot}_{z}(\alpha)\right)$. The rotated moment vector $\left({ }^{k} \boldsymbol{M}_{c}\right)$ can be obtained as

${ }^{k} \boldsymbol{M}_{c}=\operatorname{Rot}_{z}(\alpha){ }^{k} \boldsymbol{M}_{\mu \mathrm{t}}$.

Using the Castigliano's Theorem, bending $\left(\left(U_{\mathrm{b} 1}\right)_{k},\left(U_{\mathrm{b} 2}\right)_{k}\right)$ and torsional $\left(\left(U_{\mathrm{t}}\right)_{k}\right)$ strain energies are given in Eqs. (14), (15) and (16), respectively. The material properties which are modulus of elasticity and shear modulus are represented as $E$ and $G$. The geometric quantities $I, J$ and $R$ are defined as area moment inertia, polar moment inertia and radius of the helical spring.

$\left(U_{\mathrm{b} 1}\right)_{k}=\int_{0}^{2 \Pi} \frac{\left({ }^{k} \boldsymbol{M}_{c, y}\right)^{2}}{2 E I} R d \alpha$

$\left(U_{\mathrm{b} 2}\right)_{k}=\int_{0}^{2 \Pi} \frac{\left({ }^{k} \boldsymbol{M}_{c, z}\right)^{2}}{2 E I} R d \alpha$

$\left(U_{\mathrm{t}}\right)_{k}=\int_{0}^{2 \Pi} \frac{\left({ }^{k} \boldsymbol{M}_{c, x}\right)^{2}}{2 G J} R d \alpha$

Summation of three strain energies give full strain energy of a single coil $\left((U)_{k}\right)$ and it is added for every coil to calculate the total strain energy of the spring $(U)$, which are given in Eqs. (17) and (18), respectively.

$(U)_{k}=\left(U_{\mathrm{b} 1}\right)+\left(U_{\mathrm{b} 2}\right)+\left(U_{\mathrm{t}}\right)_{k}$

$(U)=\sum_{k=0}^{n-1}(U)_{k}$ 
The Castigliano's Theorem states that derivatives of the strain energies equal to the deformations which are the compression distance $\Delta d_{2}$ and the 2-D bending angle $\rho$ which is projection of pitch and roll angles on 2-D plane.

$$
\begin{gathered}
\Delta d_{2}=\frac{\partial(U)}{\partial F_{\mathrm{eq}}} \\
\rho=\frac{\partial(U)}{\partial M_{\mathrm{eq}}}
\end{gathered}
$$

As a result, the nonlinear force-deformation relation or stiffness equation of the spring can be written in given form in Eq. (20).

$$
\left[\begin{array}{c}
F_{\text {eq }} \\
M_{\text {eq }}
\end{array}\right]=[\mathbf{K}]\left[\begin{array}{c}
\Delta d_{2} \\
\rho
\end{array}\right]
$$

The analysis is performed by using the Matlab Symbolic Toolbox for each $\Delta d_{2}$ (between $0^{\circ}$ and $32 \mathrm{~mm}$ with $1 \mathrm{~mm}$ increments) and $\rho$ (between 0 and $40^{\circ}$ with $1^{\circ}$ increments). The result of $2 \times 2 \mathbf{K}$ matrices are collected within a look-up table. Wire and spring radii are 1.5 and $16.5 \mathrm{~mm}$, respectively. The number of active coils are 8 and the material of the spring is ASTM A227. In the dynamic model, the algorithm uses the appropriate $\mathbf{K}$ matrix values according to the generalised coordinates. With the definition of $\mathbf{K}$ matrix as in Eq. (22), the $K_{12}$ and $K_{21}$ elements are same due to the symmetrical structure of the stiffness matrix. The simulation results for $K_{11}, K_{12}$ and $K_{22}$ elements are given in Fig. 8.

$[\mathbf{K}]=\left[\begin{array}{ll}K_{11} K_{12} \\ K_{21} K_{22}\end{array}\right]$

$K_{11}$ can be interpreted as the relation between the translational motion of the upper plate $\Delta d_{2}$ and the reaction force of the spring in the same direction. Since the stiffness matrix is symmetric, $K_{12}$ and $K_{21}$ are equal. $K_{12}$ is the relation between combined rotation $\rho$ and reaction force of the spring. Similarly, $K_{21}$ connects $\Delta d_{2}$ and the reaction moment. The combined rotation and the reaction moment are connected by $K_{22}$. According to the Fig. 8, when both roll and pitch angles are zero, the translational motion has no effect on the change of the elements of stiffness matrix. Thus, this configuration of the mechanism can be called as a singular position for stiffness. On the other hand, all of the elements of the matrix decreases with the increase in the value of $\Delta d_{2}$, which means the spring gets softer with the compression. Compared to other parameters, $K_{11}$ responds to the change of $\rho$ differently. The decrease of $K_{11}$ is obvious with the increasing values of $\rho$ while the other parameters increase. So, the bending motion softens the spring for compression effect and it gets stiffer for rotation. Although the stiffness of the mechanism is adjusted with the translational motion of the upper plate, the bending angle has more effect on the stiffness of the mechanism. As a result of this, the stiffness value depends on the configuration of the mechanism. But, it also changes with the
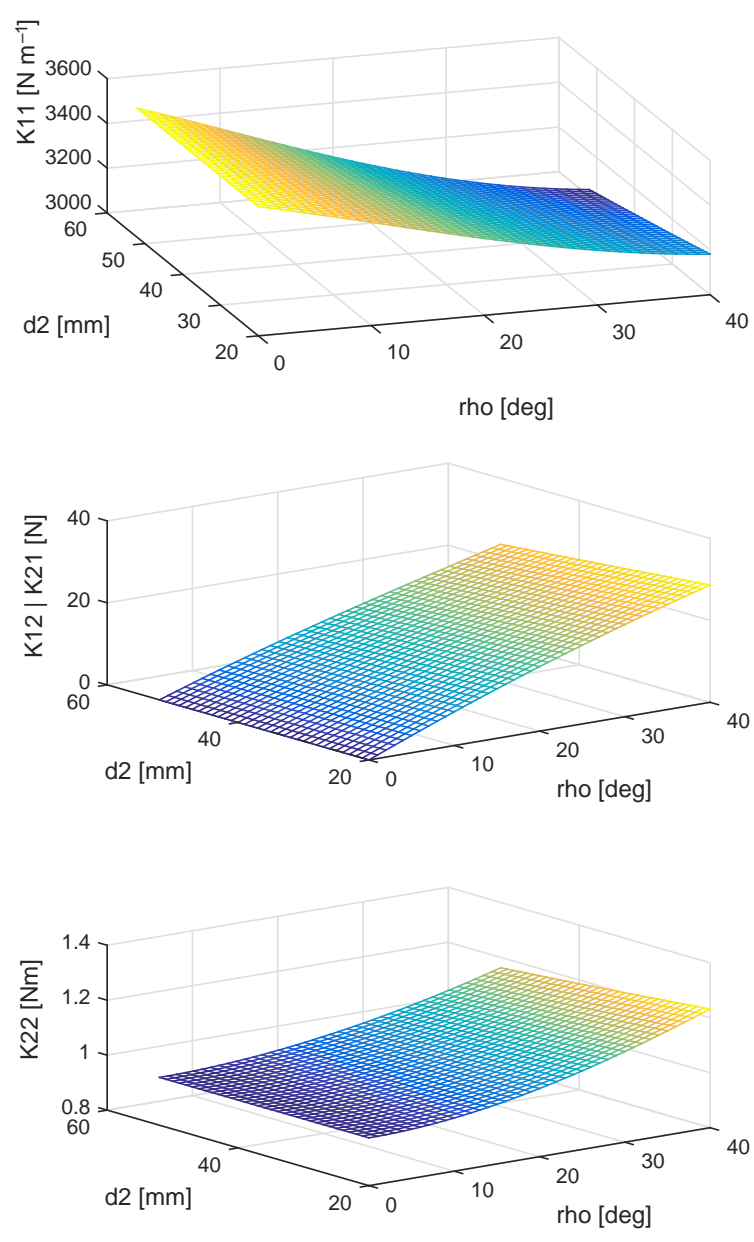

Figure 8. Stiffness matrix change with respect to bending angle and compression length.

variation of $\Delta d_{2}$ which gives variable stiffness ability to the mechanism.

Since finite element analysis (FEA) is commonly used tool for structural mechanics, this technique is used to validate the results of the stiffness analysis proposed in this study. In Fig. 9a and b, initial shape and the deformed shape of the mechanism are shown, respectively. The upper and the lower plates and the shaft are assumed to be rigid parts to isolate spring in analysis particularly and to simplify the model. Additionally, the spring is fixed to lower and upper plates. A revolute joint relation is defined between two shaft parts. Similarly, a translational joint constraint is described between the upper plate and the lower part of the shaft. A prescribed motion profile is applied to these relations as $10 \mathrm{~mm}$ translation and $40^{\circ}$ rotation. As a result, reaction forces and moments of the spring are obtained during the motion.

FEA and proposed stiffness analysis technique are compared in Fig. 10. Both translation and rotation motions are applied as a linearly increasing function, i.e. ramp function. Thus, $x$ axes of Fig. 10a and b are given as compression in millimetres and bending in degrees. The reaction forces 


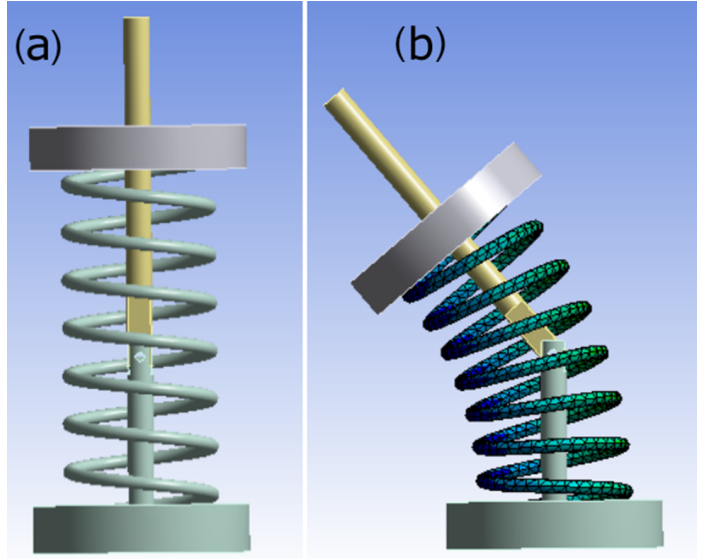

Figure 9. The models of the mechanism in finite element analysis. (a) Initial, (b) deformed.

and moments are calculated using FEA and stiffness values which are given in Fig. 8. The mean errors between FEA and the proposed method are $2.2 \%$ for reaction force and $1.5 \%$ for reaction moment. A convergence problem can be seen in the initial steps of FEA method. Moreover, quadratic function behaviour can be seen from both force and moment graphs which is a result of linearly changing stiffness values. Subsequently, the results of FEA demonstrates that linear helical spring shows a nonlinear behaviour subjected to bending and compression effects.

\subsection{Complete Dynamic Model}

The equation of motion of a serial manipulator is defined as in Eq. (23).

$$
\mathbf{M}(\boldsymbol{q}) \ddot{\boldsymbol{q}}+\mathbf{C}(\boldsymbol{q}, \dot{\boldsymbol{q}}) \dot{\boldsymbol{q}}+\mathbf{G}(\boldsymbol{q})=\mathbf{B}(\boldsymbol{q}) \boldsymbol{T}+\boldsymbol{F}_{\mathrm{s}}(\boldsymbol{q})
$$

The vector $\boldsymbol{q}$ is the generalized coordinates vector $\left[\theta, \phi, d_{2}\right]^{\mathrm{T}}$, $\dot{\boldsymbol{q}}$ and $\ddot{\boldsymbol{q}}$ are the velocity and acceleration vectors, respectively. $\mathbf{M}$ is the mass matrix, $\mathbf{C}$ includes Coriolis and centrifugal forces and $\mathbf{G}$ is the gravity matrix. There are no motors connected joints directly. Cable tensions are taken into account with a mapping matrix $\mathbf{B}$ which is given in Eq. (24). $\boldsymbol{F}_{\mathrm{S}}$ is the generalized force vector of helical spring and $\boldsymbol{s}_{i}$ is the unit vector on the $i$ th cable direction. The variables $P_{i, x}$, $P_{i, y}, P_{i, z}$ are the positions of connection points of upper plate and each cable.

$$
\begin{aligned}
& \mathbf{B}(\boldsymbol{q})= \\
& \quad\left[\begin{array}{ccc}
s_{1, z} & s_{2, z} & s_{3, z} \\
-P_{1, z} s_{1, y}+P_{1_{y}} s_{1, z} & -P_{2, z} s_{2, y}+P_{2 y} s_{1, z} & -P_{2, z} s_{2, y}+P_{2 y} s_{2, z} \\
P_{1, z} s_{1, x}-P_{1_{x}} s_{1, z} & P_{2, z} s_{2, x}-P_{2 x} s_{2, z} & P_{2, z} s_{2, x}-P_{2 x} s_{2, z}
\end{array}\right]
\end{aligned}
$$

\section{Experiments and Results}

In this section, the implementation of the proposed mechanism is explained. First, the mechanical construction and

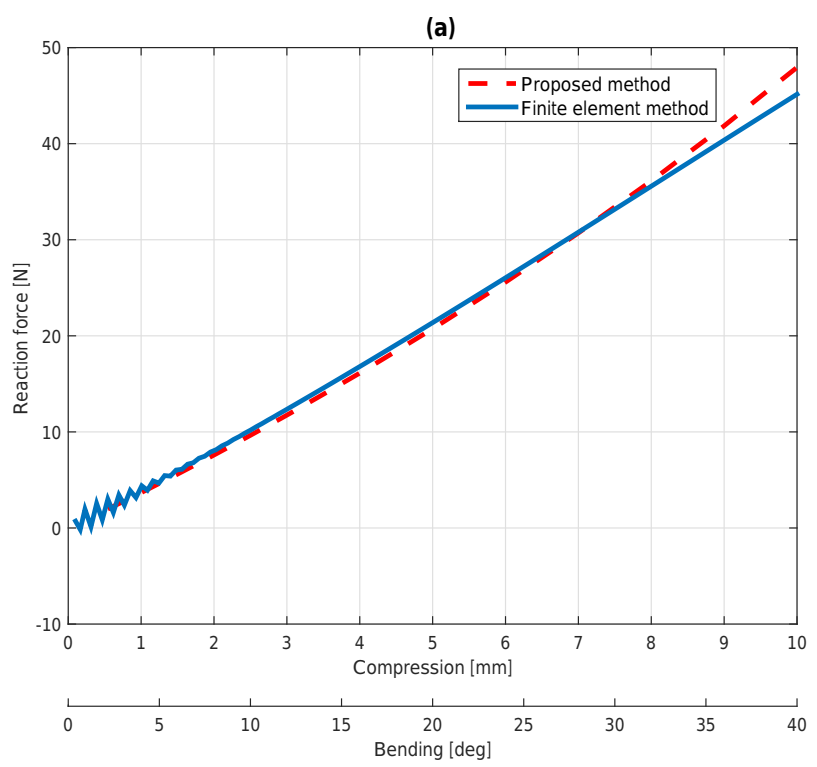

(b)

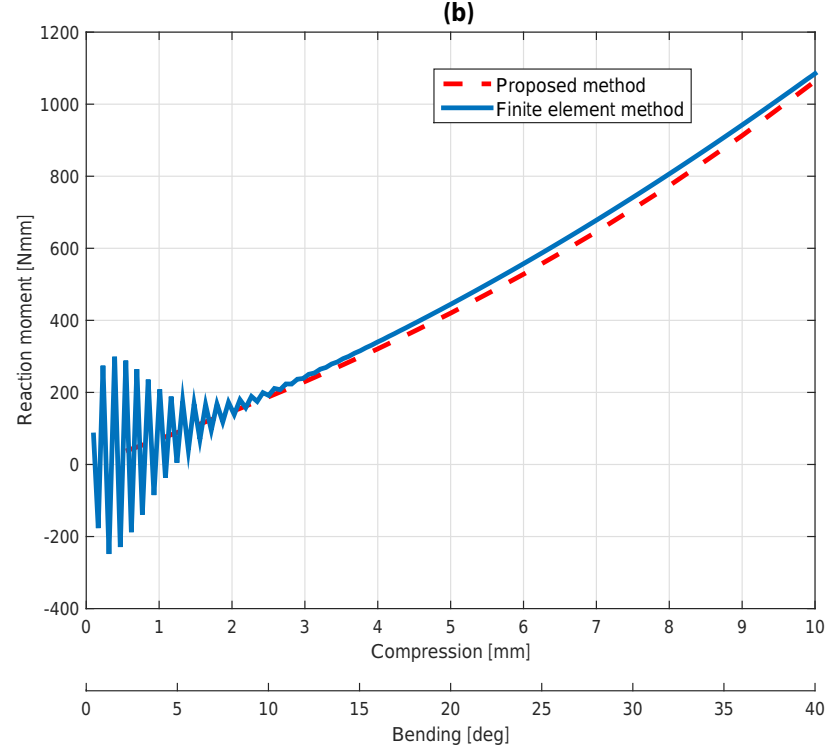

Figure 10. Behaviour of the spring under compression and bending effects. (a) Reaction force. (b) Reaction moment.

components are introduced. Then control structure, sensors and the software are given. In the experimental results section, the mechanism performs a controlled motion where the parameters are measured to validate the kinematic and dynamic models. The implemented mechanism is used as humanoid neck in UMAY project (Boyraz et al., 2013).

\subsection{Experimental Setup}

The mechanism is constructed in order to perform validation of both kinematic and dynamic models. Aluminum (7075) is used in upper and lower plates. The shafts are made of 


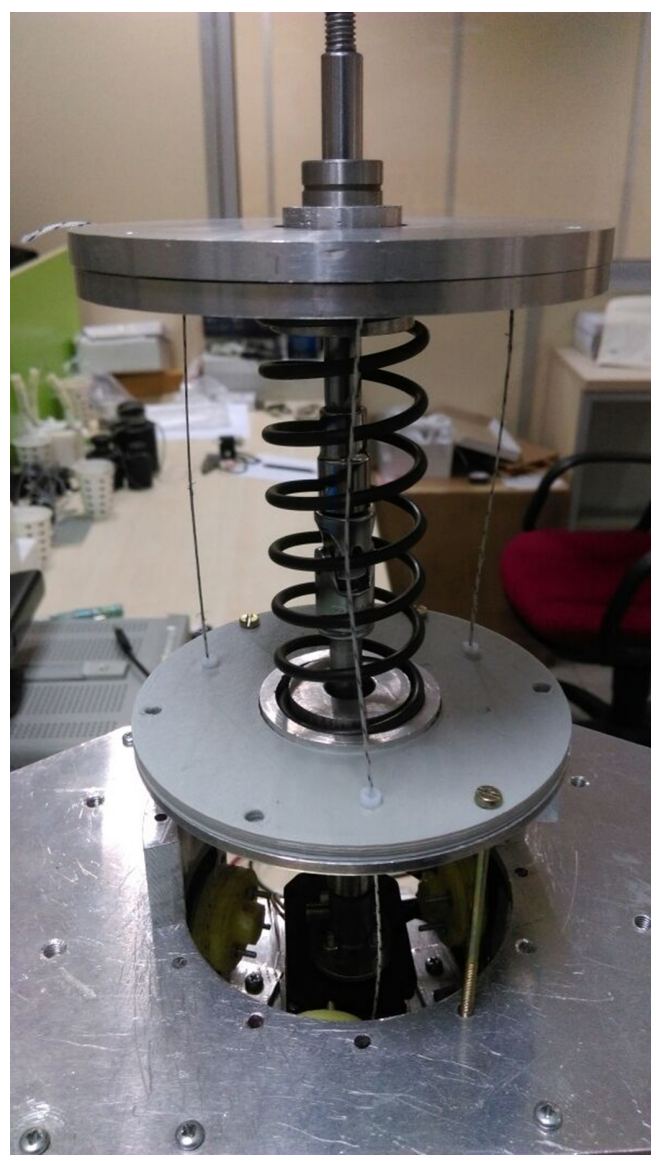

Figure 11. UMAY neck mechanism.

chromed steel (AISI E 52100). Four Robotis Dynamixel MX28 motors, which has $2.5 \mathrm{Nm}$ stall torque at $12 \mathrm{~V}$, are used. One of the motors are directly connected to shaft in order to perform yaw motion. Other three motors are connected to the cables with $14 \mathrm{~mm}$ radius capstans. Fishing lines (with $0.5 \mathrm{~mm}$ radius) are used as cable material which have a $100 \mathrm{~N}$ payload capacity. The friction force between the lower plate and the cables are reduced using PTFE tubes (with $2 \mathrm{~mm}$ inner radius) in $O_{1}, O_{2}$ and $O_{3}$ points. A picture of the implemented mechanism is given in Fig. 11 and a video is presented in the Supplement. The video is composed of randomly chosen pitch and roll motion combinations. Although the translational motion of the upper plate in zero positions of pitch and roll angles neither affects the stiffness nor the orientation, it is illustrated in the video to emphasize the motion. Three cables are pulled equal amount in length to obtain pure translational motion in the video.

\subsection{Results}

The experimental setup is equipped with Razor 9 DOF inertial measurement unit (IMU) which is attached on the upper plate along the $x$ axis. The IMU is used to collect the ori-

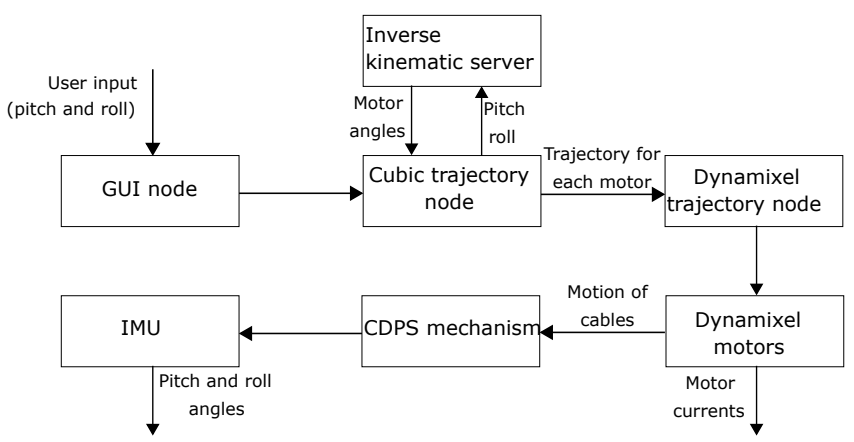

Figure 12. Control structure used in experiments.

entation data of output shaft in pitch and roll axes. IMU is used to create a truth table to validate the orientation outputs. The only feedback devices are the encoders of Dynamixel motors.

The whole position control structure used in experiments is programmed within Robot Operating System (ROS) which can be seen in Fig. 12. It is worth noting that the control scheme considers only kinematics of the mechanism. A graphical user interface (GUI) node is designed to collect data from the human user. The commands are sent to cubic trajectory node which enquires necessary motor angles from inverse kinematic server. Since the aim is to validate the models, the server calculates the necessary motor angle changes with the simplified solution of inverse kinematics as explained in Sect. 3. In this solution, one of the cables are held at a constant position. Therefore, the inverse kinematic server returns with the other cable lengths and $d_{2}$ parameter while ignoring the stiffness value. The cubic trajectory node takes these arguments as input and calculates a cubic polynomial for motor angles. The total trajectory time is given as $2 \mathrm{~s}$ for each trajectory which is a feasible amount of time for given workspace and motors. The cubic trajectory of motor shaft angles are sent to Dynamixel trajectory node.

In this section, all given data are obtained from a single one of several experiments. Thus, all figures are connected to each other. The experimental results are given for pitch/roll angles and related motors. The yaw axis motion and dynamics are excluded because they do not affect the motion of the upper plate and stiffness. The experiment is performed for $80 \mathrm{~s}$ which is long enough for an arbitrary duration to validate random pitch and roll input commands. A user gives random pitch and roll angle commands using GUI which is given in detail in this section. The experimental results are presented in two categories. First, in order to validate kinematic model, orientation of the output shaft and forward kinematics are compared. Second, motor load data and the inverse dynamics solution are examined.

Kinematic results of the experiment are given in Fig. 13 for pitch and roll axes. The user command is shown as step function in figures which starts a $2 \mathrm{~s}$ cubic trajectory. Forward 

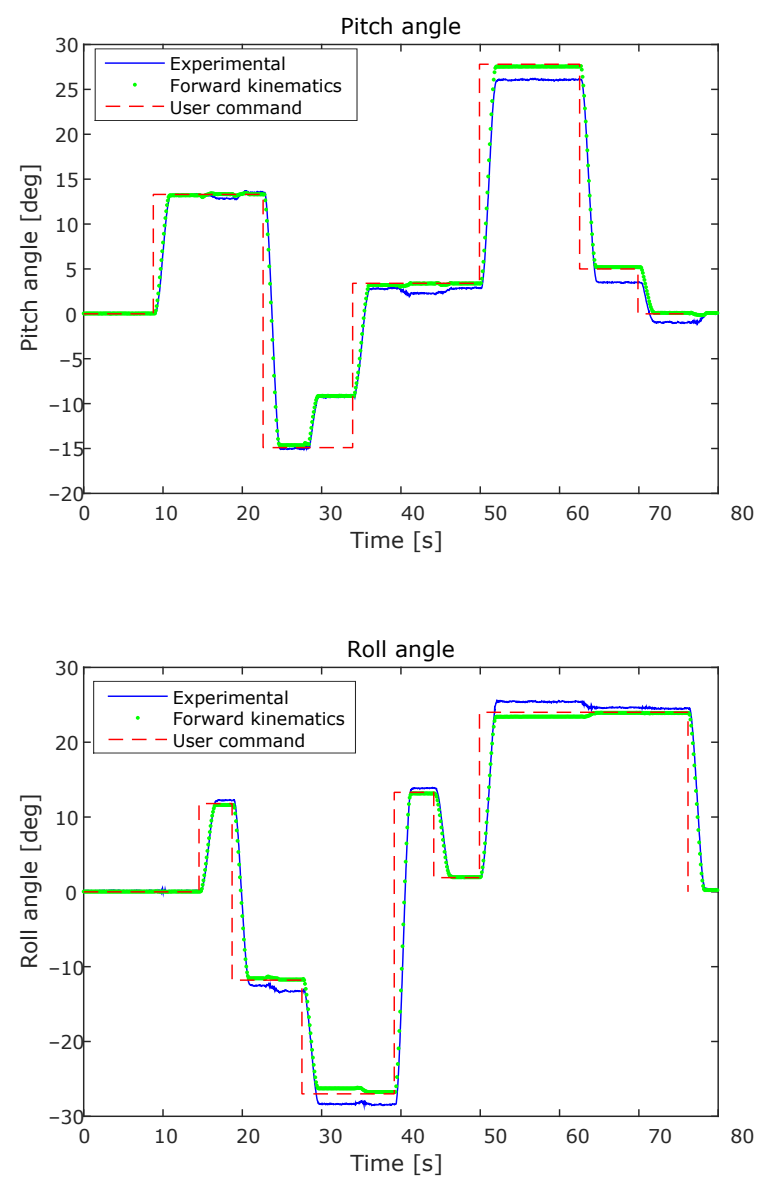

Figure 13. Angular positions of the output shaft in pitch and roll axes.

kinematic solutions are calculated using motor shaft angles which are obtained from experiment.

Inverse dynamic algorithm is performed using user commands collected from GUI in order to estimate cable tensions for each motor. Additionally, the load data is used to estimate cable tensions which is provided by Dynamixel motors and obtained from motor current values. Two cable tension estimations are compared for front cable, left cable and right cable as can be seen in Fig. 14. The general behavior of these data for each motor are similar, but there are errors in some parts of these results because of three reasons. First, the motor current measurements are very noisy due to the use of pulse width modulation of the motor low level controller. Second, neglected effects such as friction in gearbox of motor, friction between cable and PTFE parts appears difference between torque estimation from motor current and inverse dynamics model result. Finally, DC motors have no load current which is not taken into account in this estimation.
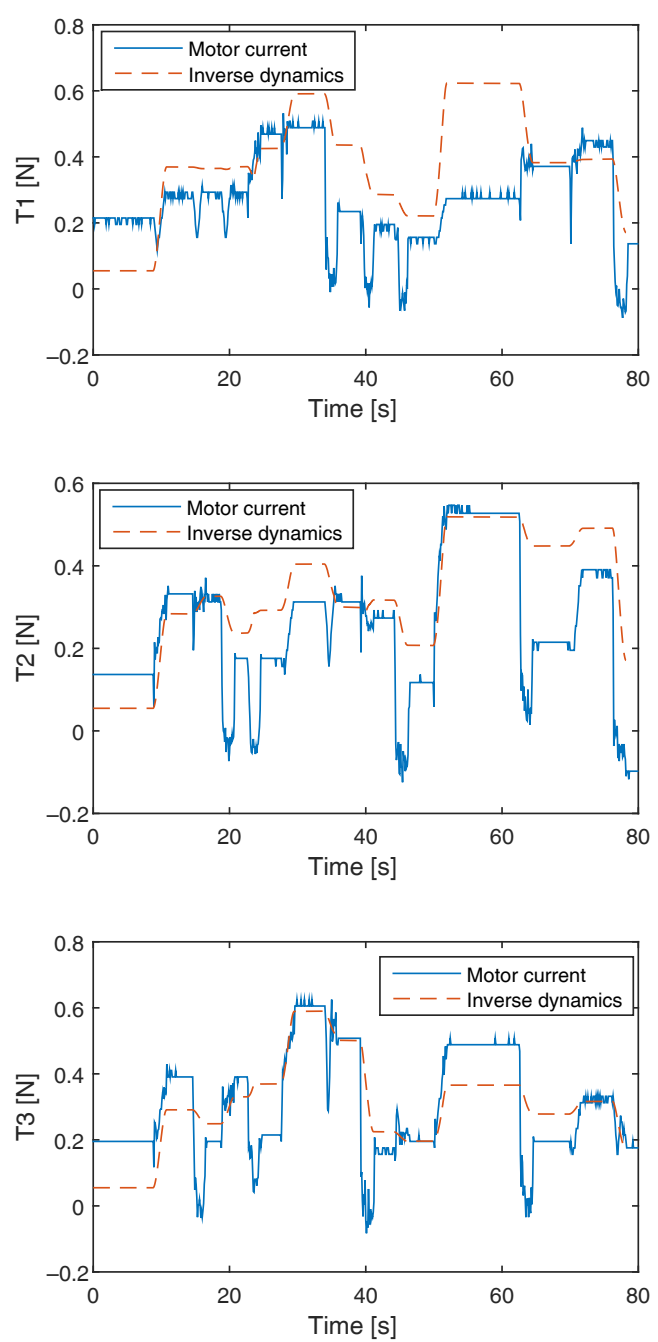

Figure 14. Cable tension estimations from inverse dynamics and motor currents.

\section{Conclusions}

A cable-driven parallel-series hybrid variable stiffness joint mechanism is presented in this paper. First, the mechanism is explained reporting advantages and disadvantages. Then, kinematic analysis of the mechanism is given considering it as a serial manipulator, simplifying the solution. By using the forward kinematics the workspace is computed. Then, dynamic model is presented. The stiffness analysis of helical spring between upper and lower plates is explained and for the spring which is used in the experimental setup, the look-up table of stiffness matrix according to compression and bending angle is calculated. To validate the results of the proposed helical spring analysis method, FEA is applied for a motion scenario and the results of both analysis are compared. 
The proposed mechanism is implemented and operated to validate the models given in the paper. Experiments are studied for kinematics and dynamics. In kinematics part, the orientation of the output shaft is compared to forward kinematics computations. It is shown that forward kinematics can predict motion profile of the output shaft. In dynamics section, two estimations of cable tensions, one from motor current and the other one from inverse dynamics calculations, are examined. It can be seen that, the results from the inverse dynamic calculations are similar to the motor current values.

In future work, the closed-loop stiffness control and hybrid position-force control techniques are going to be applied to the mechanism. With its large workspace, smooth motion profiles, lightweight mechanical structure and variable stiffness properties, the possible application area to this mechanism could be humanoid robots. It is indeed used as a humanoid neck mechanism in UMAY project. Humanoid robots interact with their environments and humans. Force interaction with humans requires safe operation conditions. After applying force control techniques, it can be used in humanoid joints where the force interaction occurs such as arms and hands in a safe manner as a result of compliant nature of the mechanism.

Data availability. All the data used in this manuscript can be obtained by requesting from the corresponding author.

\section{The Supplement related to this article is available online at doi:10.5194/ms-8-65-2017-supplement.}

Competing interests. The authors declare that they have no conflict of interest.

Acknowledgements. The project is supported by Scientific Research Projects Unit of Istanbul Technical University Young Researcher Award.

Edited by: M. Wojtyra

Reviewed by: two anonymous referees

\section{References}

Alici, G. and Shirinzadeh, B.: Topology optimisation and singularity analysis of a 3-SPS parallel manipulator with a passive constraining spherical joint, Mech. Mach. Theory, 39, 215-235, 2004.

Bennett, S. E., Schenk, R. J., and Simmons, E. D.: Active range of motion utilized in the cervical spine to perform daily functional tasks, Clinical Spine Surgery, 15, 307-311, 2002.

Boyraz, P., Yigit, C. B., and Bicer, H. O.: UMAY 1: A modular humanoid platform for education and rehabilitation of children with autism spectrum disorders, IEEE, 9th Asian Control Conference (ASCC), 1-6, 2013.

Cao, D. and Tucker, R. W.: Nonlinear dynamics of elastic rods using the Cosserat theory: modelling and simulation, Int. J. Solids Struct., 45, 460-477, 2008.

Conrad, B. L., Jung, J., Penning, R. S., and Zinn, M. R.: Interleaved continuum-rigid manipulation: An augmented approach for robotic minimally-invasive flexible catheter-based procedures, IEEE International Conference on Robotics and Automation (ICRA), 718-724, 2013.

Ferrario, V. F., Sforza, C., Serrao, G., Grassi, G., and Mossi, E.: Active range of motion of the head and cervical spine: a threedimensional investigation in healthy young adults, J. Orthop. Res., 20, 122-129, 2002.

Gao, B., Xu, J., Zhao, J., and Xi, N.: Combined inverse kinematic and static analysis and optimal design of a cable-driven mechanism with a spring spine, Adv. Robotics, 26, 923-946, 2012a.

Gao, B., Xu, J., Zhao, J., Xi, N., Shen, Y., and Yang, R.: A humanoid neck system featuring low motion-noise, J. Intell. Robot. Syst., 67, 101-116, 2012b.

Gravagne, I. A., Rahn, C. D., and Walker, I. D.: Large deflection dynamics and control for planar continuum robots, IEEE/ASME Transactions on Mechatronics, 8, 299-307, 2003.

Grioli, G., Wolf, S., Garabini, M., Catalano, M., Burdet, E., Caldwell, D., Carloni, R., Friedl, W., Grebenstein, M., Laffranchi, M., Lefeber, D., Stramigioli, S., Tsagarakis, N., van Damme, M., Vanderborght, B., Albu-Schaeffer, A., and Bicchi, A.: Variable stiffness actuators: The user's point of view, Int. J. Robot. Res., 34, 727-743, 2015.

Ham, R. V., Sugar, T. G., Vanderborght, B., Hollander, K. W., and Lefeber, D.: Compliant actuator designs, IEEE Robot. Autom. Mag., 16, 81-94, 2009.

Hollander, K. W., Sugar, T. G., and Herring, D. E.: Adjustable robotic tendon using a “jack spring”, in: Rehabilitation Robotics, IEEE, 9th International Conference on ICORR 2005, 113-118, 2005.

Jones, B. A. and Walker, I. D.: Kinematics for multisection continuum robots, IEEE T. Robot., 22, 43-55, 2006.

Kim, J. S., Jeong, J. H., and Park, J. H.: Inverse kinematics and geometric singularity analysis of a 3-SPS/S redundant motion mechanism using conformal geometric algebra, Mech. Mach. Theory, 90, 23-36, 2015.

Leech, A. R.: A study of the deformation of helical springs under eccentric loading, PhD thesis, Naval Postgraduate School, Monterey, California, 1994.

Lim, W., Yeo, S., Yang, G., and Mustafa, S.: Kinematic analysis and design optimization of a cable-driven universal joint module, in: Advanced Intelligent Mechatronics, IEEE/ASME International Conference on AIM 2009, 1933-1938, 2009.

Lim, W. B., Yang, G., Yeo, S. H., and Mustafa, S. K.: Modular Cable-Driven Robotic Arms for Intrinsically Safe Manipulation, Service Robots and Robotics: Design and Application: Design and Application, p. 274, IGI Global, Hershey PA, 2012.

Liu, X.-J., Wang, J., and Pritschow, G.: A new family of spatial 3DoF fully-parallel manipulators with high rotational capability, Mech. Mach. Theory, 40, 475-494, 2005.

Mizuuchi, I., Tajima, R., Yoshikai, T., Sato, D., Nagashima, K., Inaba, M., Kuniyoshi, Y., and Inoue, H.: The design and control of the flexible spine of a fully tendon-driven humanoid "Kenta", 
IEEE/RSJ International Conference on Intelligent Robots and Systems, 3, 2527-2532, 2002.

Nori, F., Jamone, L., Sandini, G., and Metta, G.: Accurate control of a human-like tendon-driven neck, IEEE, 7th IEEE-RAS International Conference on Humanoid Robots, 371-378, 2007.

Potkonjak, V., Svetozarevic, B., Jovanovic, K., and Holland, O.: The puller-follower control of compliant and noncompliant antagonistic tendon drives in robotic systems, Int. J. Adv. Robot. Syst., 8, 143-155, 2011.

Tunay, I.: Spatial continuum models of rods undergoing large deformation and inflation, IEEE T. Robot., 29, 297-307, 2013.

Vanderborght, B., Albu-Schaeffer, A., Bicchi, A., Burdet, E., Caldwell, D. G., Carloni, R., Catalano, M., Eiberger, O., Friedl, W., Ganesh, G., Garabini, M., Grebenstein, M., Grioli, G., Haddadin, S., Hoppner, H., Jafari, A., Laffranchi, M., Lefeber, D., Petit, F., Stramigioli, S., Tsagarakis, N., Van Damme, M., Van Ham, R., Visser, L. C., and Wolf, S.: Variable impedance actuators: A review, Robot. Auton. Syst., 61, 1601-1614, 2013.
Walker, I. D.: Continuous backbone "continuum" robot manipulators, ISRN Robotics, 2013, 726506, doi:10.5402/2013/726506, 2013.

Wendlandt, J. M. and Sastry, S. S.: Design and control of a simplified Stewart platform for endoscopy, Proceedings of the 33rd IEEE Conference on Decision and Control, 1, 357-362, 1994.

Woehrmann, M., Doerbaum, M., Ponick, B., and Mertens, A.: Design of a fully actuated electromagnetic bending actuator for endoscopic applications, in: Innovative Small Drives and MicroMotor Systems, GMM/ETG Symposium, 9, 1-6, 2013.

Yang, G., Lin, W., Pham, C., and Yeo, S. H.: Kinematic design of a 7-DOF cable-driven humanoid arm: a solution-in-nature approach, IEEE/ASME International Conference on Advanced Intelligent Mechatronics, Proceedings, 444-449, 2005. 